\title{
Speciation of presumptive viridans streptococci from early onset neonatal sepsis
}

\author{
P. W. J. WEST, RIMA AL-SAWAN*, H. A. FOSTER†, QUDSIYA ELECTRICWALA, ALEYAMMA \\ ALEX and D. PANIGRAHI
}

Department of Medical Laboratory Science, Kuwait University, *Department of Paediatrics, Farwania Hospital, Kuwait and †Department of Biological Sciences, University of Salford, Salford M5 4WT

\begin{abstract}
Twenty isolates resembling viridans streptococci, 16 from blood and four from gastric aspirates, from 17 cases of early onset neonatal sepsis were identified by the API20 Strep, Rapid ID 32 Strep and conventional tests plus hydrolysis of methylumbelliferyl glycoside substrates. Nineteen of the isolates were identified as species of viridans streptococci and one as a Leuconostoc sp. Ten of the isolates were Streptococcus oralis, three $S$. mitis biotype 1 , two $S$. mitis biotype 2 and one each of $S$. sanguis, $S$. vestibularis, $S$. salivarius and $S$. intermedius. The Rapid ID 32 Strep and conventional plus methylumbelliferyl tests gave the same species identity for 17 of the isolates. $S$. intermedius was identified by the Rapid ID 32 Strep as $S$. constellatus and $S$. salivarius as $S$. equinus, with $S$. salivarius at lower probability. The API20 Strep failed to identify $S$. vestibularis and identified $S$. salivarius as $S$. defectivus. The absence of certain critical tests, including urea hydrolysis, does not allow the API20 Strep to identify all the currently recognised species of viridans steptococci. The species distribution was unexpected and the incidence of $S$. oralis and other viridans streptococci in vaginal swabs from prenatal patients is being investigated further.
\end{abstract}

\section{Introduction}

Infection is a major cause of mortality and morbidity in neonates and Streptococcus spp. have frequently been implicated as pathogens [1,2]. S. pyogenes predominated in the first half of the century [3] to be replaced by $S$. agalactiae in the 1960s [4]. In the 1980s several hospitals reported increased isolations of so called 'viridans streptococci', which in some cases exceeded the number of isolations of S. agalactiae during the same period [2,4]. An earlier study of neonatal septicaemia reported on 99 positive blood cultures obtained from 94 neonates. Specimens from 17 babies yielded viridans streptococci, a total which was exceeded only by Escherichia coli and Klebsiella pneumoniae and was higher than S. agalactiae, which was isolated from the blood cultures of six neonates [5]. The latter study and others [2, 4] have been mainly clinical and species identification has either not been included, or details of the identification methods used have not been given. Adams and Faix [6] reported six cases of neonatal infection, all early onset, and stated the identification method used. These workers identified their isolates as

Received 19 Dec. 1997; accepted 8 Feb. 1998.

Corresponding author: Dr P. W. J. West.
S. mitis by the API Rapid Strep; however, the biochemical characteristics of the isolates were not reported.

Beighton and colleagues [7] showed that $S$. oralis and to a lesser extent $S$. mitis are the species of viridans streptococci most frequently isolated from the blood cultures of adult neutropenic cancer patients. They attribute this to the ability of these organisms to obtain nutrients from host-derived glycoproteins by virtue of their glycolytic and proteolytic abilities [8, 9]. Beighton et al. [10] have developed an identification scheme for viridans streptococci based on hydrolysis of methylumbelliferyl-linked glycosidase substrates together with tests for arginine and aesculin and urea hydrolysis and carbohydrate fermentation. Kilian et al. [11] also proposed a classification scheme based on hydrolysis of $\alpha$-naphthyl- and nitrophenollinked substrates and a methylumbelliferyl substrate for sialidase activity, in addition to conventional tests.

In the report by Beighton et al. [7] there was poor agreement between results obtained with the API20 Strep, (bioMérieux, La Balme les Grottes, France) and Beighton's conventional and methylumbelliferyl substrate methods, as $S$. oralis was not included in the version of the API20 Strep profile register used. 
However, $S$. oralis is included in the most recent version (V5.1) that has been issued by bioMérieux. BioMérieux have also introduced the Rapid ID 32 Strep which includes more tests for glycosidases and proteases than the API20 Strep, and identifies viridans streptococci according to nomenclature which more closely fits currently accepted taxonomy [10-12].

Until 1995 we identified neonatal streptococci by the API20 Strep system. We have now converted to the Rapid ID 32 Strep, and during the transition period isolates were profiled with both systems. Isolates were also tested by conventional tests based on those of Waitkins et al. [13, 14] and with methylumbelliferyl substrates as described by Beighton et al. [10].

\section{Materials and methods}

\section{Bacterial strains}

Twenty isolates from blood cultures or gastric aspirates of 17 neonates aged $\leqslant 2$ days, reported as presumptive 'viridans streptococci' on initial isolation, were investigated. Storage of clinical isolates and control strains was as reported previously [14].

All biochemical tests were performed with cultures grown on Columbia Blood Agar (Oxoid CM331) with sheep blood (Saudi Prepared Media) 5\% incubated anaerobically for $18-24 \mathrm{~h}$. All cultures and biochemical tests were incubated at $37^{\circ} \mathrm{C}$. Inoculation of conventional tests was as described previously [14].

\section{Biochemical tests}

All the organisms were catalase negative, gram-positive cocci or coccobacilli, resistant to optochin.

Arginine hydrolysis was performed by a modification of the method of Waitkins et al. [13, 14]. Aesculin hydrolysis was tested both in Blood Agar Base (Oxoid CM271) and Brain Heart Infusion Agar (BBL 11065) supplemented with aesculin $0.1 \%$ and ferric chloride $0.05 \%$ and dispensed in 2-ml volumes in 5-ml bottles; results were recorded daily for 5 days. Acetoin production (Voges-Proskauer test) was determined by the method of Waitkins et al. [13]. Dextran and levan production were tested on Trypticase Soy Agar (Oxoid CM131) with sucrose 5\% incubated in air for 3 days, and examined for glossy adherent colonies. Greening on chocolate agar was tested on blood agar base with sheep blood $5 \%$ heated for $15 \mathrm{~min}$ at $80^{\circ} \mathrm{C}$ before pouring. Hydrogen peroxide production was detected with $1-\mathrm{cm}$ squares of blotting paper impregnated with diaminobenzidine $0.1 \% \mathrm{w} / \mathrm{v}(50 \mu \mathrm{l})$ and glucose $1 \%(50 \mu \mathrm{l})$. Chocolate agar plates were heavily inoculated with test strains and incubated for $6 \mathrm{~h}$ in air. Paper squares with diaminobenzidine and glucose were placed on the growing cultures and on uninoculated control plates. A drop of hydrogen peroxide $3 \%$ was dropped on to the surface of the chocolate agar close to a paper as a positive control. The plates were re-incubated in air and strains that produced a black zone around the paper were recorded as positive for hydrogen peroxide production. Urease activity was tested in Urease Broth (BBL 21098) which was inoculated to give a turbidity of 1.0 McFarland units and incubated for $24 \mathrm{~h}$. Vancomycin susceptibility was performed on Mueller Hinton Agar (Oxoid CM337) with lysed sheep blood 5\%. Streptococcal grouping was carried out with the Avipath-Strep kit (Omega Diagnostics). Hydrolysis of methylumbelliferyl substrates was performed according to the method of Beighton et al. [10], except that 50- $\mu 1$ volumes of substrate and bacterial suspension were used. API20 Strep and Rapid ID 32 Strep methods (BioMérieux), were performed according to the manufacturer's instructions. API results were read with APILAB plus software V5.1 for the API20 Strep and V1.1 for the Rapid ID 32 Strep.

\section{Results}

All the organisms tested were isolated from blood or gastric aspirate cultures of babies aged $\leqslant 2$ days. The source of the organisms and results obtained with conventional tests and methylumbelliferyl substrates are shown in Table 1, together with species identification. The isolates were numbered sequentially after identification to simplify presentation and discussion of the results. Isolates from the same baby that showed biochemical differences were given the same serial number, and recorded as $A$ and $B$ variants. Weak reactions in the Voges-Proskauer (VP) test and for aesculin hydrolysis in conventional media are shown, but were considered as negative for the purpose of assigning species identity.

API20 Strep and Rapid ID 32 Strep profiles and identities derived from the profile number are shown in Table 2. In generating the profile number weak VP and aesculin reactions were recorded as negative.

Ten of the isolates (1-9A) were identified as $S$. oralis based on their reactions in conventional tests and production of $\beta$-galactosidase, sialidase and $\mathrm{N}$-acetyl$\beta$-glucosaminidase. All the isolates showed alkaline phosphatase activity in the API20 Strep. The same species identity was obtained for all the isolates with the Rapid ID 32 Strep at probabilities of $\geqslant 98.2 \%$, and with the API20 Strep for six isolates at probabilities of $\geqslant 99.0 \%$. The four exceptions with the API20 Strep were one isolate that was aesculin positive, identified as $S$. oralis with $89.6 \%$ probability, two isolates identified as $S$. sanguis at low probability and one identified as $S$. mitis. The strains identified as $S$. sanguis were recorded as negative for starch fermentation (0270450), but had this reaction been positive (0270451) would have been identified as S. oralis at $99.0 \%$ probability. The strain identified as $S$. mitis was recorded $\alpha$-galactosidase negative 


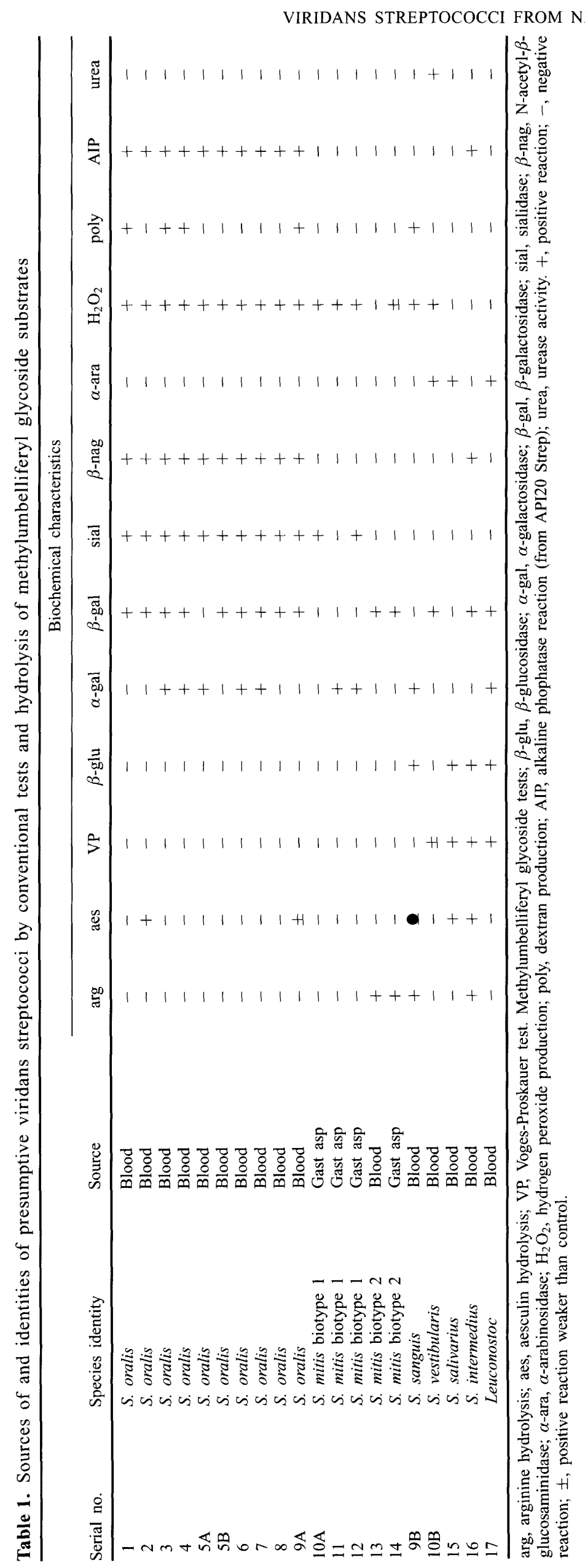


Table 2. Speciation of presumptive isolates of viridans streptococci by the Rapid ID 32 Strep and API20 Strep systems

\begin{tabular}{|c|c|c|c|c|}
\hline \multirow[b]{2}{*}{ Serial no. } & \multicolumn{2}{|c|}{ Rapid ID 32 Strep } & \multicolumn{2}{|c|}{ AP20 Strep } \\
\hline & Profile no. & Identity $(\%)$ & Profile no. & Identity $(\%)$ \\
\hline 1 & 46076643100 & S. oralis $(99.8)$ & 0270451 & S. oralis $(99.0)$ \\
\hline 2 & 66076643120 & S. oralis $(99.8)$ & 4270451 & S. oralis $(89.6)$ \\
\hline 3 & 46076643100 & S. oralis (99.9) & 0270441 & S. oralis (99.9) \\
\hline 4 & 46052643120 & S. oralis $(99.9)$ & 0270441 & S. oralis $(99.9)$ \\
\hline $5 \mathrm{~A}$ & 66072443121 & S. oralis $(99.4)$ & 0270450 & S. sanguis (54.5) \\
\hline $5 B$ & 46056643100 & $S$. oralis $(99.9)$ & 0270451 & $S$. oralis $(99.9)$ \\
\hline 6 & 46072443100 & S. oralis $(99.2)$ & 0270451 & S. oralis $(99.9)$ \\
\hline 7 & 66076641120 & S. oralis $(99.1)$ & 0270451 & S. oralis $(99.0)$ \\
\hline 8 & 46076643120 & S. oralis $(99.9)$ & 0270450 & $S$. sanguis $(54.5)$ \\
\hline $9 \mathrm{~A}$ & 02052463120 & S. oralis $(98.2)$ & 0070441 & S. mitis (88.1) \\
\hline $10 \mathrm{~A}$ & 02052043100 & S. mitis 1 (50.6) & 0240440 & $S$. sanguis (43.4) \\
\hline 11 & 00012441100 & S. mitis 1 (99.1) & 0040400 & S. mitis $2(97.0)$ \\
\hline 12 & 02052443100 & S. mitis (68.7) & 0240440 & S. sanguis (43.4) \\
\hline 13 & 50016441100 & S. mitis 2 (99.9) & 0051401 & S. mitis 1 (99.5) \\
\hline 14 & 50076441100 & $S$. mitis $2(82.7)$ & 0051401 & $S$. mitis 1 (99.5) \\
\hline $9 \mathrm{~B}$ & 30032441120 & S. sanguis $(99.6)$ & 4041411 & S. sanguis (86.6) \\
\hline $10 \mathrm{~B}$ & 60036401102 & S. vestibularis $(94.5)$ & 0050410 & $S$. oralis $(55.1)$ \\
\hline 15 & 20022341110 & S. equinus (68.9) & 5040211 & Listeria (38.8) \\
\hline 16 & 54023001110 & S. constellatus (99.1) & 5061010 & $S$. intermedius $(88.0)$ \\
\hline 17 & 62054003101 & Leuconostoc (99.9) & 1210450 & $S$. defectivus (52.6) \\
\hline
\end{tabular}

(0070401), but had $\alpha$-galactosidase been positive $(0270441)$ would have been identified as $S$. oralis at $99.9 \%$ probability. Five isolates $(10 \mathrm{~A}-14)$ were identified as $S$. mitis by the Rapid API 32 Strep. Three of these (10A, 11 and 12) met the criteria for $S$. mitis given by Beighton et al. [10] and are recorded as $S$. mitis biotype 1 , following the terminology of Kilian et al. [11]. Two of these isolates were identified as $S$. mitis 1 by the Rapid ID 32 Strep, but as $S$. mitis 2 by the API20 Strep. Isolate 12 was identified as $S$. mitis by the Rapid ID 32 Strep at $68.7 \%$ probability, and as S. sanguis (43.4\%) by the API20 Strep, which would have identified $S$. oralis at $95.6 \%$ had the starch hydrolysis result been positive. Two isolates (13 and 14) were identified as $S$. mitis biotype 2 according to the terminology of Kilian et al. [11]. These strains were arginine positive and aesculin negative, but were excluded from $S$. sanguis, $S$. parasanguis, $S$. gordonii and $S$. crista (tufted fibril group) by the absence of $\mathrm{N}$ acetyl- $\beta$-glucosaminidase, $\beta$-glucosidase, $\alpha$-galactosidase, $\alpha$-fucosidase, $\beta$-fucosidase or $\alpha$-arabinosidase activity [10]. They were identified as $S$. mitis 2 by the Rapid ID 32 Strep, but as $S$. mitis 1 by the API20 Strep. Strain 9B was a typical S. sanguis, and was identified as this species by all the methods used. Isolate $10 \mathrm{~B}$ showed a typical biochemical pattern for $S$. vestibularis in conventional tests (weak VP and urease positive) as well as $\alpha$-arabinosidase activity [15], and was identified as this species by the Rapid ID 32 Strep. However, the API20 Strep software does not include $S$. vestibularis, and the organism was identified as $S$. oralis with low probability. Isolate 15 was identified as $S$. salivarius as it was $\beta$-glucosidase and $\alpha$-arabinosidase positive, arginine negative and produced levan on sucrose $5 \%$ agar. The organism was identified as $S$. equinus $(68.9 \%)$ by the Rapid ID 32 Strep and Listeria sp. $(38.8 \%)$ by the API20 Strep system. Both identities were rejected as the organism was negative for Lancefield group $D$ antigen and catalase activity. The commercial systems offered $S$. salivarius at lower probability for this isolate (22.1 and $12.5 \%$ respectively). Isolate 16 was identified as $S$. intermedius by conventional methods and methylumbelliferyl substrates and by the API20 Strep, but as $S$. constellatus $(99.1 \%)$ by the Rapid ID 32 Strep. These species plus $S$. anginosus were previously often reported as ' $S$. milleri' [16], but were separated by differences in hydrolysis of methylumbelliferyl glycoside substrates $[17,18]$. Isolate 17 was identified as a Leuconostoc sp. at high probability $(99.9 \%)$ by the Rapid ID 32 Strep and this identity was accepted because the organism was vancomycin resistant in addition to being pyrrolidonylarylamidase and leucine aminopeptidase negative in both API systems [19]. The API20 Strep for this isolate gave $S$. defectivus at $52.6 \%$ and Leuconostoc sp. at $36.3 \%$ probability.

\section{Discussion}

All the organisms identified in this study were from early onset cases of suspected neonatal sepsis. Therefore, we believe that the organisms were acquired from the mother by vertical transmission. Ten of the 16 blood culture isolates were typical $S$. oralis as defined by Beighton et al. [10]. Although $S$. oralis is the species most frequently reported from adult neutropenic patients we have not noted any reports of isolations of this species from neonates, and did not recognise it before 1995, because it was not included in versions of the API20 Strep profile register prior to V5.1, in which $S$. oralis is included. All the isolates of $S$. oralis were alkaline phophatase positive, a result that concurred with the description by Kilian et al. [11]. 
A further five isolates were identified as $S$. mitis based on the descriptions of Beighton et al. [10] and Kilian et al. [11], although $S$. mitis biotype 2 isolates in the present study were negative for $\mathrm{N}$-acetyl- $\beta$-glucosaminidase and $\beta$-glucosidase activity with methylumbelliferyl substrates. Kilian et al. [11] proposed $S$. mitis biotype 2 for arginine positive strains from his collection which had $S$. mitis-type cell-wall composition (Lys-direct peptidoglycan and ribitol teichoic but lacking rhamnose). The cell-wall composition of the isolates was not investigated in the present study. Both API systems include two $S$. mitis types, referred to as $S$. mitis 1 and $S$. mitis 2 in the list of species identified. $S$. mitis 1 is given as arginine negative and S. mitis $299 \%$ arginine positive in the Rapid ID 32 Strep literature; however, the API20 Strep profile register includes arginine positive and negative variants in both $S$. mitis 1 and $S$. mitis 2 . Hence an isolate may be identified as $S$. mitis 1 by one API kit and as $S$. mitis 2 by the other. Both Spigelblatt et al. [2] and Adams and Faix [6] reported S. mitis from neonatal blood cultures and all the cases documented by Adams and Faix [6] were early onset. S. salivarius and $S$. sanguis isolates were reported by Spigelblatt et al. [2].

Members of the 'S. milleri group' have been reported from neonates and from vaginal specimens of the mothers of infected babies [16]. S. vestibularis has been reported from the blood culture of an adult patient [20], but not to our knowledge from neonates. Members of the genus Leuconostoc have been reported from cases of sepsis, including a late onset case in a study of neonatal meningitis [21, 22].

The spectrum of organisms found in the present study, with the exception of $S$. oralis, is similar to that recorded by Spigelblatt et al. [2] who identified 10 of 19 neonatal blood culture isolates as $S$. mitis, eight as single isolates and two in mixed cultures with another Streptococcus sp., and also identified $S$. sanguis and $S$. salivarius. Some of the isolates identified as $S$. mitis by Spigelblatt et al. [2] could have been $S$. oralis, as although this species was first proposed by Bridge and Sneath in 1982 [23] and an emended description was given by Kilian et al. in 1989 [11], it was rarely reported from clinical specimens before publication of the identification scheme of Beighton et al. [10] in 1991 and its introduction into API profile registers.

There was excellent agreement in species identity between the Rapid ID 32 Strep and conventional plus methylumbelliferyl substrate tests, with agreement to species level for 17 of 19 streptococcal isolates, provided that two $S$. mitis biotypes were accepted. $S$. intermedius was identified as $S$. constellatus, a closely related species $[17,18]$. The Leuconostoc sp. was also correctly identified. The only major discrepancy was in the identification of the isolate that was identified as $S$. salivarius based on conventional and rapid methods; however, both API systems did offer $S$. salivarius at lower probability. The API20 Strep system performed less satisfactorily than the Rapid ID 32 Strep, as it does not contain certain critical tests such as urea and $\mathrm{N}$-acetyl- $\beta$-glucosaminidase which are essential to identify all the currently recognised species of viridans streptococci.

The predominance of $S$. oralis was a novel finding and the occurrence of this and other species of viridans streptococci in vaginal swabs taken from prenatal patients is currently being investigated.

\section{References}

1. Gladstone IM, Ehrenkranz RA, Edberg SC, Baltimore RS. A ten-year review of neonatal sepsis and comparison with the previous fifty-year experience. Pediatr Infect Dis $J$ 1990; 9: $819-825$.

2. Spigelblatt L, Saintonge J, Chicoine R, Laverdiere M. Changing pattern of neonatal streptococcal septicemia. Pediatr Infect Dis 1985; 4: 56-58.

3. Nyhan WL, Fousek MD. Septicemia of the newborn. Pediatrics 1958; 22: 268-278.

4. Broughton RA, Krafka R, Baker CJ. Non-group D alphahemolytic streptococci: new neonatal pathogens. $J$ Pediatr 1981; 99: 450-454.

5. Al-Sawan RMZ, Jesuraj L, West P, Al-Saleh Q. Bacterial septicemia in Farwania neonatal unit Kuwait. J Kwt Med Assoc 1997; 29: 291-296.

6. Adams JT, Faix RG. Streptococcus mitis infection in newborns. $J$ Perinatol 1994; 14: 473-478.

7. Beighton D, Carr AD, Oppenheim BA. Identification of viridans streptococci associated with bacteraemia in neutropenic cancer patients. $J$ Med Microbiol 1994; 40: 202-204.

8. Homer KA, Kelley S, Hawkes J, Beighton D, Grootveld MC Metabolism of glycoprotein-derived sialic acid and n-acetyglucosamine by Streptococcus oralis. Microbiology 1996; 142: $1221-1230$.

9. Homer KA, Whiley RA, Beighton D. Proteolytic activity of oral streptococci. FEMS Microbiol Lett 1990; 67: 257-260.

10. Beighton D, Hardie JM, Whiley RA. A scheme for the identification of viridans streptococci. J Med Microbiol 1991; 35: $367-372$.

11. Kilian M, Mikkelsen L, Henrichsen J. Taxonomic study of viridans streptococci: description of Streptococcus gordonii $\mathrm{sp}$. nov. and emended descriptions of Streptococcus sanguis (White and Niven 1946), Streptococcus oralis (Bridge and Sneath 1982), and Streptococcus mitis (Andrewes and Horder 1906). Int $J$ Syst Bacteriol 1989; 39: 471-484.

12. Freney J, Bland S, Etienne M, Desmonceaux M, Boeufgras JM, Fleurette J. Description and evaluation of the semiautomated 4hour Rapid ID 32 Strep Method for identification of streptococci and related genera. J Clin Microbiol 1992; 30: $2657-2661$.

13. Waitkins SA, Ball LC, Fraser CA. A shortened scheme for the identification of indifferent streptococci. J Clin Pathol 1980; 33: $47-52$.

14. West PWJ, Foster HA, Electricwala Q, Alex A. Comparison of five methods for the determination of arginine hydrolysis by viridans streptococci. $J$ Med Microbiol 1996; 45: 501-504.

15. Whiley RA, Hardie JM. Streptococcus vestibularis sp. nov. from the human oral cavity. Int $J$ Syst Bacteriol 1988; 38: $335-339$.

16. Raymond J, Bergeret M, Francoual C, Chavinie J, Gendrel D. Neonatal infection with Streptococcus milleri. Eur $J$ Clin Microbiol Infect Dis 1995; 14: 799-801.

17. Whiley RA, Beighton D. Emended descriptions and recognition of Streptococcus constellatus, Streptococcus intermedius, and Streptococcus anginosus as distinct species. Int J Syst Bacteriol 1991; 41: 1-5

18. Whiley RA, Fraser H, Hardie JM, Beighton D. Phenotypic differentiation of Streptococcus intermedius, Streptococcus 
constellatus, and Streptococcus anginosus strains within the "Streptococcus milleri" group. J Clin Microbiol 1990; 28: 1497-1501.

19. Facklam R, Pigott N, Franklin R, Elliott J. Evaluation of three disk tests for indentification of enterococci, leuconostocs, and pediococci. J Clin Microbiol 1995; 33: 885-887.

20. Cunliffe NA, Jacob AJ. Streptococcus vestibularis bacteraemia. $J$ Infect 1997; 34: 85

21. Facklam R, Elliott JA. Identification, classification, and clinical relevance of catalase-negative gram-positive cocci, excluding the streptococci and enterococci. Clin Microbiol Rev 1995; 8: 479-495.

22. Friedland IR, Snipelisky $M$, Khoosal $M$. Meningitis in a neonate caused by Leuconostoc sp. J Clin Microbiol 1990; 28: 2125-2126.

23. Bridge PD, Sneath PHA. Streptococcus gallinarum sp. nov. and Streptococcus oralis sp. nov. Int J Syst Bacteriol 1982; 32: $410-415$. 